\title{
The relationship between Raynaud's phenomenon and Helicobacter pylori
}

\author{
Alat I \\ Department of Cardiovascular Surgery, Afyonkarahisar State Hospital, Afyonkarahisar, Turkey. \\ ilkeralat@hotmail.com
}

\begin{abstract}
BACKGROUND: This study was aimed to reveal whether there is a relationship between Raynaud Phenomenon (RP) and Helicobacter Pylori (HP).

MATERIAL AND METHODS: Seventy-nine patients, who had been referred to outpatient clinic with Raynaud in the last 9 years were retrospectively screened. Of these, 29 patients with access to their data and who had HP screening tests were included in the study.

RESULTS: HP direct antigen was found in feces in only one of 29 patients. When we compared the results of this study to the results of previous literature, it was observed that the patients admitted with RP symptoms by a gastroenterology outpatient clinic had a higher incidence for HP (+) scanning than the patients admitted with RP symptoms by a cardiovascular surgery policlinic (CVSOC).

CONCLUSION: Although previous literature reports that HP leads to RP in the group of patients referred to other outpatient clinics, the same relation is very low in the Raynaud patient group in CVSOC. Patients admitted with RP in the CVSOC shouldn't be prescribed empirical antibiotic therapy for the eradication of HP. However, as the appropriate antibiotic regimen can resolve Raynaud symptoms in the presence of a HP(+) test, it makes this scanning rational for all symptomatic patients (Tab. 1, Fig. 1, Ref. 23). Text in PDF www.elis.sk. KEY WORDS: Raynaud syndrome, Raynaud phenomenon, Helicobacter pylori, vasculitis, gastritis.
\end{abstract}

\section{Introduction}

Even if Raynaud Syndrome (RS), first described in 1862 by Maurice Raynaud (1) was later divided into different definitions like RS or Raynaud Phenomenon (RP) or primary (PRP) and secondary RP (SRP) (2), it still keeps its obscurity despite the evolving invasive and non-invasive diagnostic and therapeutic possibilities of cardiovascular surgery. As the result, unknown etiology is causing the unsatisfactory outcomes in the treatment of PRP or SRP, unfortunately.

Establishing the true prevalence of RP has been hampered by a number of factors including populations, climates, genetic and environmental influences (2). Previous literatures reported that the pathophysiology of RP is complex and multifactorial, involving the endothelium, neuroreceptor expression, and locally produced mediators that affect vascular responses $(2,3)$. Additionally, especially for the etiology of SRP, some different clinical disorders like hypothyroidism with high thyroid-stimulating hormone levels (4) or vascular toxicity associated with anti-neoplastic agents (5) or some rheumatic diseases like systemic lupus erythematosus, Sjogren's syndrome and scleroderma(2) were also considered re-

Department of Cardiovascular Surgery, Afyonkarahisar State Hospital, Afyonkarahisar, Turkey

Address for correspondence: I. Alat, Dr, Afyonkarahisar Devlet Hastanesi, Kalp ve Damar Cerrahisi Servisi, Afyonkarahisar, Turkey.

Phone: +90.5325492475 sponsible for Raynaud's symptoms. Likewise, according to previous literature $(6,7)$, Helicobacter Pylori (HP) can be a possible factor leading to RP. These reports insist that the eradication of HP with a suitable antibiotic therapy ameliorates the symptoms of RP totally or resolves them partially at least $(6,7)$.

In this retrospective study, the existence of HP in the patients referred to the cardiovascular surgery outpatient clinic (CVSOC) with the symptoms of RP was examined and besides, the role of HP on the occurrence of RP and the need for HP scanning and the indications for the eradication of HP in the patients with RP symptoms have been discussed.

\section{Material and methods}

\section{Patients}

Seventy-nine patients, who applied to the author's outpatient clinic and were diagnosed with RP in the last 9 years were examined retrospectively. An ethical approval was obtained from the institutional board and authorities. Out of 79 patients, 50 patients were excluded from the study because their hospital records could not be accessed or HP scanning was not done. So, the findings of 29 patients were enrolled in this study.

\section{Disease diagnosis and HP scanning}

Anamnestic statements of the patients, the inspection after the cold exposure test, physical examinations and the evaluation of the arterial system of the upper and lower extremities with a 

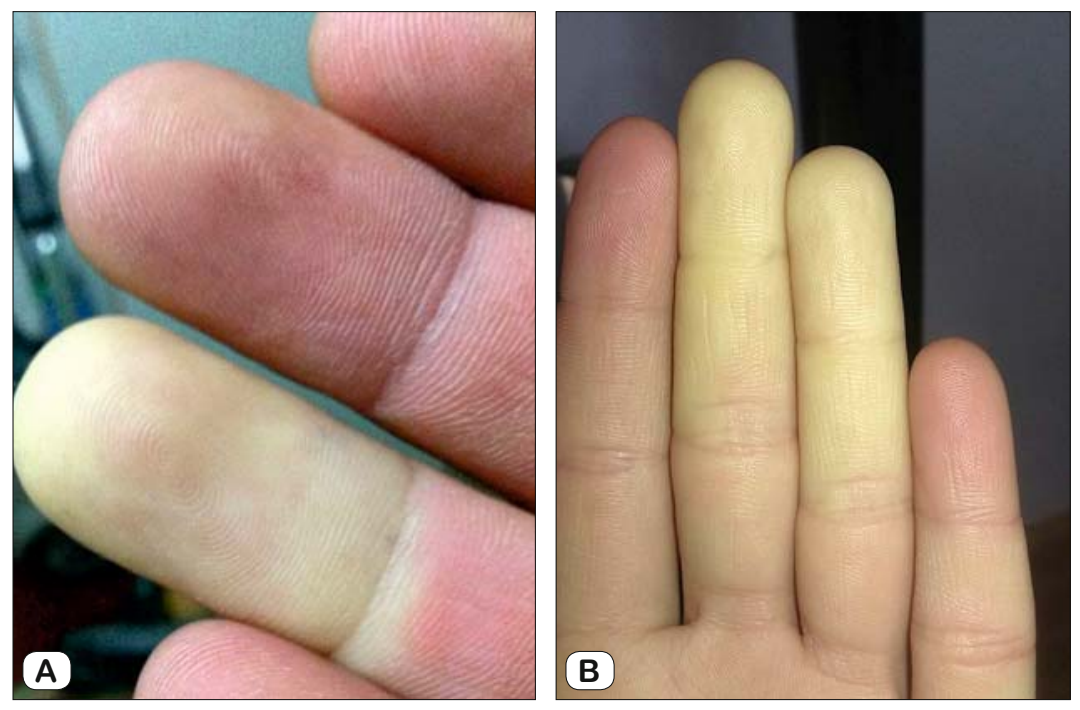

Fig. 1. Findings of a male (A) and a female (B) patient with Raynaud's phenomenon activated by exposure to cold. Note the apparent pale and yellowing in the distal phalanges of the fingers.

colored Doppler ultrasonography (CDU) were all used for the diagnosis of RP.

HP scanning was done by looking for HP direct antigens in feces. (Laboquick, Helicobacter Pylori Antigen Test, In Vitro Diagnostic Test, Labex Engineering Ltd. Sofia, Bulgaria)

\section{Additional laboratory analyses}

Hemogram, anti-streptolysin O (ASO) and C-reactive protein (CRP) levels of the patients were also noted.

\section{Other demographic findings}

In addition to the sex and age of the patients, the existence of diabetes mellitus (DM), smoking habit, gastric complaints or the existence of any gastric diagnosis, occupational predominance (especially vibratory works) were also examined.

\section{Statistics}

Test of a single proportion and binomial test were used for the statistical analyses and any $p$ value less than 0.05 was considered statistically significant.

\section{Results}

Ten patients were male and 19 patients were female. The statistical results of the ages of the patients were as followed: min: 14 years, max: 53 years, mean: 28.61 years, median: 25 years, SD: 11.40 .

After a cold exposure, changing in the color of the fingers is seen as an example for a male patient in Figure 1A and for a female patient in Figure 1B.

Cumulative data of the patients were summarized in Table 1.

It was seen that four patients had a job related to vibration. One of these patients was a sculptor and was using some vibra- ting tools to shape the marble. Interestingly, 6 patients had a job related to rubber or caoutchouc materials.

DM was not a misleading factor in terms of interpreting the results. It was observed in only a one case.

About half of the patients (n: 16, $55 \%$ ) had no smoking history.

Although there were 12 patients with some gastric complaints or diseases, there was only a one case with a positive fecal HP direct antigen. This case had a normal level of white blood cells although he had a positive fecal HP direct antigen.

The level of eosinophils were changing in 10 patients in different directions, although 19 patients had normal eosinophil levels. Although the previous literature $(8,9)$ reported that hypereosinophilic conditions may be associated with RP or vasculitis, in this retrospective study eosinophils were found to be high in some cases, and in some cases low or normal. The level of eosinophils in the patient, who had a positive fecal HP direct antigen, was lower than normal.

Pathological CDU findings were obtained in 8 patients, although the rest of them were normal on ultrasound. Two of eight patients had pathological CDU findings in their both upper and lower extremities. So, totally 10 different extremities had positive CDU findings in 29 patients. Positive CDU findings included monophasic or biphasic blood flows, increased peripheral vascular resistance and tinning in the calibration of the vessels. Occlusions of the arteries were not observed in any patient.

None of the ulcers or necrosis or gangrenous changes were observed in any of the patients. No patient underwent surgical operations like sympathectomy.

All of the patients were prescribed acetylsalicylic acid, pentoxyphylline and a peripheral arterial vasodilator agent. No complete healing was achieved in any patient. Mild or moderate re-

Tab. 1. Cumulative data of the patients.

\begin{tabular}{llc}
\hline Parameters & & Numbers \\
\hline \multirow{2}{*}{ Sex } & Male & 10 \\
\cline { 2 - 3 } Contact & Female & 19 \\
\hline Smoking & Vibration & 4 \\
\cline { 2 - 3 } Diabetes Mellitus & Rubber & 6 \\
\hline Gastric Complaint & & 13 \\
\hline Pathological & Upper Extremity & 1 \\
\cline { 2 - 3 } CDU Findings & Lower Extremity & 12 \\
\hline \multirow{2}{*}{ Eosinophil } & Low & 7 \\
\cline { 2 - 3 } & High & 3 \\
\hline CRP & & 2 \\
\hline Fecal Helicobacter Pylori Direct Antigen & 1 \\
\hline CDU - Colored Doppler Ultrasonography, CRP - C Reactive Protein
\end{tabular}

CDU - Colored Doppler Ultrasonography, CRP - C Reactive Protein 
coveries were observed in the clinical symptoms or complaints of the patients.

In the HP $(+)$ case, an eradication therapy for HP (amoxicillin + clarithromycin + proton pump inhibitor for 14 days) was added into his prescription together with other medications above. This eradication therapy was administered in two successive cycles. Next, his control HP scanning was negative. After the therapy, his complaints and clinical findings almost completely resolved.

In regard to HP scanning in patients admitted with the RP diagnosis in a CVSOC, when compared the number of the HP (+) patients to the number of the HP (-) patients, there was a statistically significant difference between the groups $(\mathrm{p}<0.05)$. In another saying, the number of the HP (+) RP patients was very low among the whole RP patients admitted in a CVSOC.

On other hand, when compared the percentage (1 patient, 3 $\%)$ of the number of the HP (+) RP patients in this study to the percentage $(75 \%)$ of the HP $(+)$ RP patients in previous literature $(6,7)$, there was a statistically significant difference between the groups $(\mathrm{p}<0.05)$. In other words, the patients admitted with RP symptoms by a gastroenterology outpatient clinic had a higher incidence for $\mathrm{HP}(+)$ scanning than the patients admitted with RP symptoms by a CVSOC.

\section{Discussion}

RS, first described in 1862 by Maurice Raynaud (1) was then divided into different definitions like RS or RP or PRP and SRP (2). Despite the current technologies and innovations in medicine, the absolute mechanism playing the role in the ethiopathological steps of RP has not been elucidated yet. The fact that the etiology cannot be determined with a certain degree of clarity also leads to the fact that no satisfactory results were achieved in the treatment of RP. Perhaps the main reason for keeping this disease unknown might be the indifference attitude of the scientists or doctors, who were capable of even working inside of an atherosclerotic plaque. Researchers are unfortunately not as interested in Raynaud as in other cardiovascular events. When considering the time elapsed from 1862 until the present, the fact that a real improvement related to RP was not obtained, can be a significant evidence that today's doctors are uninterested in this issue. Moreover, when considering the developments in cardiovascular surgery in the same 150-year- period, starting from scratch and extending to the current applications like minimal invasive surgeries, robotic surgeries, transcatheter applications, it can be thought that we, the doctors have been very unconcerned with Raynaud. This apathy leads to failure in the treatment of RP unfortunately. Because of these reasons, the need to concentrate and do research more on this disease should come into prominence.

According to previous literature, although the pathophysiology of RP is complex and multifactorial, involving the endothelium, neuroreceptor expression, and locally produced mediators that affect vascular responses $(2,3)$, on the other hand, HP can be only a single reason inducing the symptoms of RP and the eradication of HP with a suitable antibiotic therapy can ameliorate the symptoms of RP totally $(6,7)$.
Even if the HP is not the only factor in the development of $\mathrm{RP}$, if it's really shown that it has a certain percentage, it will be a significant success to provide a complete healing in a special group of patients by easy eradication of this microbe with a suitable antibiotic regime.

Under the lights of these thoughts, in this retrospective study, the existence of HP in the patients referred to the outpatient clinic of the author of this manuscript with the symptoms of RP was examined and besides, the role of HP on the occurrence of RP and the need for HP scanning and the indications for the eradication of HP in the patients with RP symptoms have been discussed from the point of a cardiovascular surgeon's view.

When the related manuscripts are examined, it's possible to see that HP is associated with coronary atheroma (10), vasculitis $(11)$, inflammation $(12,13)$, ischemic cardiac $(10,14)$ or cerebrovascular diseases (15). As an indicator of inflammation, HP causes an increase in C-reactive protein (CRP) (12), besides there are also findings regarding the development of autoimmune diseases and gastritis due to HP (16).

When studying the patients enrolled in this study, there was no increase in the CRP values of the patients. CRP was found to be within normal limits even in the single HP $(+)$ patient. The fact that CRP was not high in any patient in this study proves that not only the factors triggering the immune system, but also many different factors may be responsible for the development of RP. In other words, negative CRP of RP patients in this study can be considered as an indirect evidence that RP is a multifactorial disease that can develop with many different causes.

As a matter of fact, those people, who work in vibratory jobs or are exposed to industrial products such as rubber are also known to have RP-like clinical symptoms $(17,18)$. When the patients of this article were examined, it was seen that 4 of 29 patients (13.8 $\%$ ) are occupied with vibratory jobs. However, it is unfortunately not very effective to detect the presence of vibratory professions in the etiology of RP. Because, patients generally regard that any request including a change of their professions is a meaningless and impossible request. At this point, the query of the existence of HP in the investigation of RP etiology is much more important. Because, even the possibility that RP might have been developed secondarily to a microbe that can be completely eradicated makes the patient look forward to hear the results of HP scanning.

Considering the cases in this article, the fact that $\mathrm{HP}(+)$ was detected in only one case out of 29 seems to be contradictory to previous literature, but it has to be clarified by different explanations. Firstly, when previous literature was examined, it was seen that HP scanning was done with the tests named " $13 \mathrm{C}$ urea breath test". Whereas, HP scanning was done by looking for HP direct antigens in feces in this study. The differences in the positive HP results between the manuscripts might be due to the differences in HP scanning techniques. This means that the specificity and sensitivity of these measurement methods should be determined in addition. Unfortunately, according to current literature (19-23), there is no consensus on which diagnostic HP scanning methods is superior. Each one of them has been associated with advantages and disadvantages. Although some of the articles, as 
the one printed in 2014 (23), stated that bacterial culture from the gastric biopsy is the gold standard technique, or as the one printed in 2014 (20), stated that polymerase chain reaction based diagnosis may be considered as the gold standard, according to another article, printed in 2015 (22), none can be considered as a single gold standard in clinical practice. Articles say that urea breathing test and stool antigen test are most widely used non-invasive tests (22). However, they also state that the stool antigen detection method, although specific, is usually associated with a poor sensitivity. The $13 \mathrm{C}$ urea breath test is believed to be specific, but with a present revelation of the fact that stomach is colonized by many other urease producing bacteria makes it questionable (20). Additionally, Best and co-workers reported (21) that the thresholds used for these tests were highly variable and they were unable to identify specific thresholds that might be useful in clinical practice. They reported they need further comparative studies of high methodological quality to obtain more reliable evidence of relative accuracy between the tests. In the article of Gasbarrini et al, in which 25 RP cases were evaluated, positive HP was found in 20 of 25 cases (80\%) (6). In another report of the same author evaluating 46 RP cases, the same screening test was used and in this report, 36 cases (78\%) were found to have HP positivity (7). HP positivity was detected in only one of the 29 cases (3\%) in this study. However, it is not a correct comment to think that direct antigen measurement in the feces mentioned in this article is inferior to the method in other articles. Because, the actual problem between the reports is not the differences in the sensitivity or specificity of the tests. The explanation of this difference should be done in a different way. Another reason for that might be the differences in the admission of the patients.

When the articles of these researchers are examined, it seems that they are the doctors in the department of internal medicine. For this reason, it is natural that high HP positivity can be seen in their patients, who were admitted with RP due to gastric complaints. On the other hand, patients with RP, who have applied to a cardiovascular surgeon's polyclinic are very likely to have very different etiologies. For this reason, a very low rate of HP positivity should be accepted as a natural outcome in the outpatient clinic of a cardiovascular surgeon. The low HP positivity in this study ( $3 \%)$ is exactly related to this subject.

This result also provides an answer to the following important question: Should HP eradication therapy be empirically applied to the patient admitted with RP in the CVSOC?

The presence of HP positivity in most or all of the RP cases admitted with gastric complaints in gastroenterology or internal medicine outpatient clinics indicates that HP has a significant percentage of the causative agents of gastritis. However, there are very different groups of patients in the regard to the etiology of RP patients, who were referred to the CVSOC. Thus, while most of the RP patients in the gastroenterology or internal medicine policlinic may be directly admitted with a positive HP scanning, very few of the patients in the CVSOC will be HP-positive. For these reasons, empirical antibiotic treatment of patients with $\mathrm{RP}$, who were referred to the CVSOC will not comply with the "Rational Antibiotic Usage" principles. On the other hand, if it is assumed that the etiopathogenesis of RS or RP is obscure and, therefore, a complete healing cannot usually be achieved in the treatment, and also, if previous literature is taken into consideration that the eradication of HP is possible in $83 \%$ of HP positive RP cases and besides if $17 \%$ of them are considered to have completely resolved their RP symptoms, it will be appropriate to routinely perform HP scanning during RP symptomatic cases referred to the CVSOC. HP eradication therapy should be applied to HP positive cases only. Even if a single HP positive event is caught, it will be a major source of hope for physicians and patients because to be capable of removing all RP symptoms by 100 percent in this case will be possible.

\section{Study limitations}

As mentioned in the discussion section, since it is not yet known what the gold standard technique should be in relation to HP scanning, it may be more appropriate to conduct further prospective researches in which different techniques are used together.

Additionally, the fact that previous antibiotic use has not been fully identified is one of the limitations of this study. In prospective studies to be planned for future, previous antibiotic use of the cases should be questioned in terms of diversity, dose, and interval.

\section{Conclusion}

When the findings of this retrospective study are combined with the findings of previous literature, the following conclusions can be reached:

1. It should be noted that HP may play a role in the etiopathogenesis of RS or RP.

2. It is not appropriate to empirically prescribe HP eradication therapy for all of the patients admitted with RP findings in a CVSOC.

3. Nevertheless, it's important to perform HP screening tests in the patients admitted with RP symptoms in a CVSOC. In particular, the presence of accompanying gastric complaints should be questioned and HP scanning in this patient group should be done more carefully.

4. Prospective studies based on serological and immunohistochemical evaluations should be planned to explain the relationship between RS or RP and HP. This article is believed to be an important guide in planning such works.

\section{References}

1. Belch JJ. The phenomenon, syndrome and disease of Maurice Raynaud. Br J Rheumatol 1990; 29: 162-167.

2. Wigley FM. Raynaud's phenomenon. Curr Opin Rheumatol 1993; 5: 773-784.

3. Yetkin U, Gurbuz A. Current approach to Raynaud's Phenomenon. Turk Gogus Kalp Dama 2002; 10 (1): 56-62.

4. Latiwish AM, Feher J, Baraczka K, Racz K, Kiss R, Glaz E. Remission of Raynaud's phenomenon after $\mathrm{L}$-thyroxine therapy in a patient with hypothyroidism. J Endocrinol Invest 1992; 15 (1): 49-51. 
5. Doll DC, Yarbro JW. Vascular toxicity associated with antineoplastic agents. Semin Oncol 1992; 19 (5): 580-596.

6. Gasbarrini A, Serricchio M, Tondi P, De Luca A, Franceschi F, Ojetti VV et al. Raynaud's Phenomenon and Helicobacter Pylori Infection. Int J Angiol 1998; 7 (4): 307-309.

7. Gasbarrini A, Massari I, Serricchio M, Tondi P, De Luca A, Franceschi $\mathbf{F}$ et al. Helicobacter pylori eradication ameliorates primary Raynaud's phenomenon. Dig Dis Sci 1998; 43 (8): 1641-1645.

8. Jang KA, Lim YS, Choi JH, Sung KJ, Moon KC, Koh JK. Hypereosinophilic syndrome presenting as cutaneous necrotizing eosinophilic vasculitis and Raynaud's phenomenon complicated by digital gangrene. Br J Dermatol 2000; 143 (3): 641-644.

9. Jandus P, Bianda N, Alerci M, Gallino A, Marone C. Eosinophilic vasculitis: an inhabitual and resistant manifestation of a vasculitis. Vasa 2010; 39 (4): 344-348. doi: 10.1024/0301-1526/a000060.

10. Ossei-Gerning N, Moayyedi P, Smith S, Braunholtz D, Wilson JI, Axon AT et al. Helicobacter pylori infection is related to atheroma in patients undergoing coronary angiography. Cardiovasc Res. 1997; 35 (1): $120-124$.

11. Lidar M, Lipschitz N, Langevitz P, Barzilai O, Ram M, Porat-Katz BS et al. Infectious serologies and autoantibodies in Wegener's granulomatosis and other vasculitides: novel associations disclosed using the Rad BioPlex 2200. Ann N Y Acad Sci 2009; 1173: 649-657.

12. Jackson L, Britton J, Lewis SA, McKeever TM, Atherton J, Fullerton D et al. A population-based epidemiologic study of Helicobacter pylori infection and its association with systemic inflammation. Helicobacter 2009; 14 (5): 108-113.

13. Shen L, Matsunami Y, Quan N, Kobayashi K, Matsuura E, Oguma K. In vivo oxidation, platelet activation and simultaneous occurrence of natural immunity in atherosclerosis-prone mice. Isr Med Assoc J 2011; 13 (5): 278-283.

14. Strandberg TE, Tilvis RS, Vuoristo M, Lindroos M, Kosunen TU. Prospective study of Helicobacter pylori seropositivity and cardiovascular diseases in a general elderly population. BMJ 1997; 314: 1317-1318.
15. Markus HS, Mendall MA. Helicobacter Pylori infection: a risk factor for ischaemic cerebrovascular disease and carotid atheroma. J Neurol Neurosurg Psychiatry 1998; 64 (1): 104-107.

16. Smyk DS, Koutsoumpas AL, Mytilinaiou MG, Rigopoulou EI, Sakkas LI, Bogdanos DP. Helicobacter pylori and autoimmune disease: Cause or bystander. World J Gastroenterol 2014; 20 (3): 613-629.

17. Nilsson T, Wahlström J, Burström L. Hand-arm vibration and the risk of vascular and neurological diseases - A systematic review and meta-analysis. PLoS One 2017; 13; 12 (7): e0180795. doi: 10.1371/journal. pone.0180795.

18. Gardiner GA Jr, Tan A. Repetitive blunt trauma and arterial injury in the hand. Cardiovasc Intervent Radiol 2017; 40 (11): 1659-1668. doi: 10.1007/s00270-017-1702-5.

19. Lehours P. Actual diagnosis of Helicobacter pylori infection. Minerva Gastroenterol Dietol 2018 Apr 12. doi: 10.23736/S1121-421X.18.02494-7.

20. Patel SK, Pratap CB, Jain AK, Gulati AK, Nath G. Diagnosis of Helicobacter pylori: what should be the gold standard? World J Gastroenterol 2014: 28; 20 (36): 12847-12859. doi: 10.3748/wjg.v20.i36.12847.

21. Best LM, Takwoingi Y, Siddique S, Selladurai A, Gandhi A, Low B et al. Non-invasive diagnostic tests for Helicobacter pylori infection. Cochrane Database Syst Rev 2018 Mar 15; 3: CD012080. doi: 10.1002/14651858.CD012080.pub2.

22. Wang YK, Kuo FC, Liu CJ, Wu MC, Shih HY, Wang SS et al. Diagnosis of Helicobacter pylori infection: Current options and developments. World J Gastroenterol 2015; 21 (40): 11221-11235. doi: 10.3748/ wjg.v21.i40.11221.

23. Lopes AI, Vale FF, Oleastro M. Helicobacter pylori infection - recent developments in diagnosis. World J Gastroenterol 2014; 20 (28): 9299-9313. doi: 10.3748/wjg.v20.i28.9299.

Received November 7, 2018. Accepted July 2, 2019. 